Questions vives

\section{Questions Vives}

Recherches en éducation

$n^{\circ} 22 \mid 2014$

Questionner le curriculum en éducation physique et sportive : Quelle dynamique en contexte? Quelle autonomie?

\title{
Organisateurs de l'activité et rapport au curriculum recommandé des étudiants-stagiaires d'EPS en formation par alternance
}

What guides student teacher's activity during field experience and its

relationship to the PE national curriculum

Elisabeth Magendie

\section{OpenEdition}

12 Journals

\section{Édition électronique}

URL : http://journals.openedition.org/questionsvives/1605

DOI : $10.4000 /$ questionsvives. 1605

ISSN : $1775-433 \mathrm{X}$

Éditeur

Université Aix-Marseille (AMU)

Édition imprimée

Date de publication : 15 décembre 2014

ISBN : 978-2-912643-46-9

ISSN : 1635-4079

\section{Référence électronique}

Elisabeth Magendie, «Organisateurs de l'activité et rapport au curriculum recommandé des étudiantsstagiaires d'EPS en formation par alternance », Questions Vives [En ligne], $n^{\circ} 22$ | 2014, mis en ligne le 16 février 2015, consulté le 19 avril 2019. URL : http://journals.openedition.org/questionsvives/1605 ; DOI : 10.4000/questionsvives. 1605

Ce document a été généré automatiquement le 19 avril 2019.

\section{cc) (†)}

Questions Vives est mis à disposition selon les termes de la licence Creative Commons Attribution -

Pas d'Utilisation Commerciale - Pas de Modification 4.0 International. 


\title{
Organisateurs de l'activité et rapport au curriculum recommandé des étudiants-stagiaires d'EPS en formation par alternance
}

\author{
What guides student teacher's activity during field experience and its \\ relationship to the PE national curriculum
}

Elisabeth Magendie

\section{Introduction}

1 Cette étude s'enracine dans une visée de description des pratiques d'enseignement en tant qu'indicateurs de la production en actes du curriculum (Brière-Guenoun, 2013). Elle s'intéresse aux manières dont est mis en œuvre le curriculum effectif à partir d'analyses in situ (Crahay, Audigier \& Dolz, 2006). Son objectif est de décrire ce qui oriente et guide l'activité de quatre étudiants-stagiaires d'EPS en formation par alternance afin d'identifier ce qu'ils font des recommandations curriculaires - notamment celles préconisant un enseignement par compétences permis par une pédagogie de l'intégration (Rogiers, 2010), c'est-à-dire par la mise en place de situations complexes et de moments de réflexion sur l'action.

2 Nous envisageons de répondre aux questions de recherche suivantes: qu'est-ce qui oriente et guide l'activité des étudiants-stagiaires lorsqu'ils sont en situation de travail? En quoi les recommandations curriculaires centrées sur l'approche par compétences organisent-elles leur activité?

3 Répondre à de telles questions devrait nous permettre de mieux comprendre l'autonomie du curriculum effectif co-construit en EPS au regard des différentes recommandations dont il est l'objet, et ainsi de contribuer à l'enrichissement des connaissances sur la production en acte du curriculum. 
4 La démarche de recherche que nous avons retenue est réalisée dans une perspective de didactique professionnelle (Pastré, 2011). Elle se réfère à la théorie de la conceptualisation dans l'action (Vergnaud, 1996). Cette théorie repose sur deux idées: l'activité humaine est organisée sous la forme d'un couplage schème-situation ; le noyau central de cette organisation est composé d'invariants, de nature conceptuelle (Pastré, 2011). Notre démarche, dans ce cadre-là, s'efforce d'identifier les organisateurs qui orientent et guident l'activité des étudiants-stagiaires lors de leurs premières expériences d'enseignement.

5 Après avoir défini la problématique de la recherche et explicité son inscription théorique, nous décrivons les principes tenus pour vrais qui organisent l'activité des étudiantsstagiaires, leur activité de conception notamment. Ces résultats nous permettent ensuite de présenter les trois préoccupations centrales qui constituent les organisateurs pragmatiques dominants (Pastré, Mayen \& Vergnaud, 2006) de l'activité des étudiantsstagiaires et enfin de rendre compte de leur rapport au curriculum recommandé.

\section{Des prescriptions au « choc de la réalité »}

\subsection{Curriculum officiel et curriculum effectif}

Même s'il existe une diversité de définitions du curriculum, liées entre autres à la tension entre une approche centrée sur les disciplines et le savoir, et une approche centrée sur l'expérience de l'élève (Rey, 2010), nombreuses sont celles qui renvoient à l'idée de plan et d'organisation dont le projet est d'influer sur les processus d'enseignementapprentissage qui se déroulent en classe (Crahay, Audigier \& Dolz, 2006). Nous retiendrons, pour notre part, l'idée selon laquelle le curriculum s'organise autour de trois questions principales : que faut-il enseigner (quelles compétences)? Comment faut-il enseigner (quelles méthodes, quelles situations d'apprentissage)? Quand enseigner (quelles temporalités, quelles progressions)? (Poggi, Verscheure, Musard \& Lenzen, 2010).

7 Aborder la question du curriculum suppose également de distinguer plusieurs niveaux. Ainsi Perrenoud (1993, p. 61) montre l'existence d'un curriculum formel, réel et caché ; le curriculum caché étant «la part des apprentissages qui n'apparaît pas programmée par l'institution scolaire, du moins pas explicitement.» D'une façon générale, la plupart des chercheurs s'attachent aujourd'hui à distinguer le curriculum officiel (prescrit ou recommandé), le curriculum effectivement enseigné, et le curriculum acquis par les élèves (Rey, 2010). L'idée mise en avant est que l'existence d'un curriculum officiel ne signifie pas qu'il soit réellement enseigné. De nombreux travaux ont ainsi mis à jour les décalages existants entre le curriculum officiel et ce qui se passe effectivement dans les classes (Forquin, 2008 ; Lenoir, 2006 ; Perrenoud, 1993).

8 Mais surtout, il s'avère que l'idée première selon laquelle il y aurait trois niveaux d'emboitements successifs - du curriculum officiel jusqu'au curriculum acquis en passant par le curriculum enseigné - est fortement remise en question (Forquin, 2008). Ainsi, contrairement à ce que peut laisser supposer une telle modélisation, ce qui est enseigné en classe n'est que partiellement déterminé par les recommandations officielles (Forquin, 2008). Nombreuses sont en effet les influences susceptibles d'orienter l'activité curriculaire des enseignants. Martinand (2001, p. 21) envisage ainsi la construction des activités en classe comme le résultat d'une «composition sous influences" (sociales, 
idéologiques, politiques, pédagogiques). De tels constats ont pu être également établis dans le champ de l'EPS. Musard, Robin et Caty (2009) par exemple, ont montré que les enseignants composent les pratiques scolaires essentiellement sous l'influence de leurs collègues et des élèves. De tels résultats vont dans le sens de ceux mis en évidence par Pasco et Ennis (2009) ou Lenzen (2012). À partir d'une revue de littérature, ces auteurs ont montré le faible impact des instructions officielles sur l'élaboration des curricula par les enseignants. D'autres déterminants seraient plus influents : l'équipe EPS, les structures de formation continue, les conceptions, les orientations de valeurs ou encore les caractéristiques des élèves.

9 Nuançant cependant ces propos, Lenzen considère que les nouveaux programmes d'EPS, qui détaillent pour chaque APSA les contenus d'enseignement, sont probablement davantage pris en compte par les enseignants qu'ils ne l'étaient auparavant. D'une manière générale, Goodson (2008) invite à rester lucide sur les effets réels des prescriptions institutionnelles. Ce n'est pas selon lui parce qu'il n'y a pas de lien direct entre ce qui recommandé et ce qui est enseigné, qu'il faut renier la portée effective des programmes prescrits. Pour Goigoux (2007) également, de tels effets existent mais surtout chez les enseignants qui entrent dans le métier. L'auteur avance ainsi l'idée selon laquelle les enseignants débutants seraient particulièrement sensibles aux recommandations ou prescriptions primaires qui proviennent de l'institution scolaire, mais aussi et surtout aux prescriptions secondaires qui émanent des instituts de formation professionnelle.

Il nous semble dès lors intéressant, à l'heure où les réformes curriculaires se succèdent à un rythme soutenu, de chercher à savoir comment ces nouveaux "prescrits ", explicités dans les programmes et diffusés dans le cadre de la formation, sont appropriés par les étudiants-stagiaires qui entrent dans le métier par la voie de l'alternance.

\subsection{Le curriculum : de nouveaux « prescrits » en EPS ?}

11 Ce qui ressort des réformes curriculaires actuelles, c'est une nouvelle façon d'aborder la culture scolaire (Rey, Carette, Defrance \& Kahn, 2006). Mais ce qui saute aux yeux surtout, c'est la présence forte de l'expression « compétence », et par conséquent le passage d'une logique des savoirs à une logique des compétences.

Bien qu'il n'y ait pas de définition consensuelle de la compétence, l'idée principale à laquelle renvoie l'approche par compétences est celle de la possibilité pour un élève de savoir mobiliser un ensemble intégré de ressources, en vue de résoudre une famille de situations (Roegiers, 2004). Du point de vue de l'enseignement, cette approche s'attache à une plus grande prise en compte de l'intégration des savoirs dans une logique globale de formation (Legendre, 2008). Même si de telles orientations font l'objet de vifs débats (Crahay, 2006; Hirtt, 2009), il n'en reste pas moins que la notion de compétence est omniprésente dans le monde de l'école et de la formation (Rey, 2014) et qu'elle mérite de ce fait d'être prise en compte.

13 Comme dans les autres disciplines, les nouvelles orientations institutionnelles en EPS s'inscrivent dans une logique curriculaire organisée en termes de compétences. Il s'agit de viser le développement de compétences chez les élèves, en faisant en sorte de ne pas dissocier les savoirs que l'on juge pertinent de faire acquérir, du traitement qui en est attendu et des situations dans lesquelles ils ont vocation à être mobilisés. 

dans la plupart des académies, des groupes de réflexion visant à opérationnaliser les préconisations primaires du curriculum officiel. Dans l'académie de Bordeaux, dans laquelle sont formés les étudiants-stagiaires de notre étude, un groupe de production de ressources a élaboré des outils pour concevoir et mettre en œuvre les pratiques d'enseignement par situations complexes (Falco, 2013). L'enjeu principal des situations proposées est de convoquer simultanément les compétences attendues des programmes et les compétences transversales. Ces travaux, préconisant une pédagogie de l'intégration, sont largement diffusés dans le cadre de la formation des étudiants. Nous pouvons donc faire l'hypothèse, en référence à Goigoux (2007), qu'ils sont particulièrement prégnants chez les étudiants-stagiaires de l'académie de Bordeaux.

\subsection{L'entrée dans le métier : le « choc de la réalité »}

ombreux sont les auteurs qui se sont attachés à décrire la situation des enseignants lors de leurs premières expériences d'enseignement. Tous s'accordent sur le fait que la prise en responsabilité d'une classe génère de l'anxiété, des doutes et des remises en question qui aboutissent parfois à des échecs importants, à un sentiment d'isolement, de défaite et d'impuissance (Baillauquès \& Breuse, 1993 ; Chaliès \& Durand, 2000 ; Schmidt \& Knowles, 1995).

16 Face au "choc de la réalité », les débutants prennent rapidement conscience de la complexité du métier et renoncent alors parfois à leurs idéaux (Roux-Pérez, 2012). A la recherche de solutions fonctionnelles, voire de stratégies de survie, ils redéfinissent les tâches prescrites en distinguant une prescription maximale et exigeante et une prescription minimale, moins exigeante et réaliste (Daguzon, 2009), et recourent alors à des pratiques qui peuvent être jugées régressives par l'orthodoxie didactique ou pédagogique (Goigoux, Ria \& Toczek-Capelle, 2009).

S'inspirant de la double approche didactique et ergonomique (Robert, 2008) pour analyser les pratiques et les activités des enseignants débutants, Butlen, Charles-Pezard et Masselot (2009) montrent que ces derniers se heurtent en effet très vite aux tensions entre les logiques d'apprentissage et les logiques de socialisation. Leur principale préoccupation est l'installation d'une "paix scolaire ", c'est-à-dire à la fois une "paix sociale » et une adhésion des élèves au projet d'enseignement. Ils s'efforcent avant tout de faire accepter les règles de fonctionnement aux élèves et de les enrôler rapidement dans les tâches scolaires pour obtenir le calme. paradigme de l'action située, il ressort que l'activité des débutants se transforme peu à peu, notamment pour ceux qui sont en établissements difficiles (Ria \& Rouve, 2009 ; Ria, 2009). Après la mise en danger de leur identité professionnelle, ils tentent de reprendre en main les situations scolaires, pour se protéger et retrouver une certaine légitimité. Ils privilégient les actions efficaces de leur propre point de vue, en rejetant (provisoirement) les critères institutionnels qui leur procurent de l'inconfort (Ria, 2009). Apparaissent alors des formes d'activité à la marge des préconisations de l'institution.

19 Saujat (2004), à partir d'une approche clinique de l'activité, parvient à des conclusions similaires. Les débutants « tordent » les cadres d'exercice de leur métier (les prescriptions institutionnelles mais aussi les rapports aux élèves, à la hiérarchie, etc.) pour sortir des impasses qui «tordent» leur activité. Ils sont conduits à faire des choix qu'ils savent 
discutables sur le plan didactique mais qui renvoient pour eux à une sorte d'efficacité malgré tout. L'auteur montre, plus généralement, que les débutants ont des façons communes de travailler qui consistent à accentuer les techniques vouées à " prendre » la classe ou à la « tenir » en délaissant celles qui consistent à la « faire ». Il s'agit là d'actes faisant l'objet de préoccupations partagées par ceux qui entrent dans le métier. Ce qui conduit Saujat à parler d'un genre débutant, qui au même titre que les "genres d'activités professorale", vient s'intercaler entre le sujet et les prescriptions auxquelles il est soumis.

Les travaux conduits dans le champ de la didactique professionnelle centrés sur l'activité enseignante sont récents (Pastré, Mayen \& Vergnaud, 2006). L'enjeu est de constituer un panel d'heuristiques développées par les enseignants et des formes de rationalisation qu'ils développent (Numa-Bocage, Clauzard \& Pastré, 2012). Le travail enseignant est caractérisé par Vinatier $(2012,2013)$ comme une activité en tension entre trois registres de fonctionnement: le registre épistémique (cheminement du savoir), le registre pragmatique (conduite de la séance) et le registre relationnel (relations entre les personnes). Vinatier $(2012,2013)$ montre, à partir d'analyses centrées sur le processus de conceptualisation en acte (Vergnaud, 1996), que les débutants, lors de l'entrée dans le métier, sont confrontés à un problème récurrent: faire sa place face aux élèves, être reconnu et avoir de l'autorité sur le groupe «alors qu'ils ne sont que stagiaires». Cela signifie que les enjeux didactiques sont subordonnés à la gestion pragmatique et relationnelle de la conduite de la séance.

21 Ces quelques résultats rendent compte de ce qui organise prioritairement l'activité des débutants en classe : se débrouiller au mieux, quitte à «tordre » les prescriptions, pour obtenir la paix scolaire et «tenir » chaque jour. Selon Roux-Pérez (2012) cependant des formes de différenciation existent. La prise en main d'une classe n'est pas automatiquement une épreuve à traverser. D'ailleurs, selon cette auteure, pour plus de la moitié des stagiaires en EPS, elle se réalise, d'après eux, sans difficulté. Qu'est-ce qui organise dès lors leur activité ? N'ont-ils pas à gérer des préoccupations contradictoires? N'ont-ils pas à renoncer ou tout au moins à redéfinir «l'idéal » transmis en formation (Daguzon \& Goigoux, 2012)?

\section{Une étude inscrite dans le courant de la didactique professionnelle}

22 Afin de mieux comprendre les formes singulières d'actualisation du curriculum effectif en EPS, nous cherchons à décrire la logique de l'activité en contexte des étudiants-stagiaires à partir du cadre théorique de la didactique professionnelle (Pastré, 2011). Il nous semble en effet que cette voie de recherche, peu empruntée dans le champ de l'EPS, constitue un cadre pertinent pour l'analyse de l'activité de l'enseignant.

\subsection{L'analyse de l'activité}

23 Le cadre théorique de référence qui fonde l'analyse de l'activité est celui de la conceptualisation dans l'action (Vergnaud, 1996). L'analyse consiste à identifier les organisateurs de l'activité, c'est-à-dire les schèmes mobilisés en situation. Le schème est 
défini comme " une organisation invariante de la conduite dans une classe de situations donnée » (Vergnaud, 1996, p. 283). Il est analysable en différentes composantes :

- un but (ou plusieurs), des sous-buts et des anticipations ;

- des règles d'action, de prise d'information et de contrôle ;

- des invariants opératoires (concepts en acte et théorèmes en acte);

- des possibilités d'inférences.

Ce sont les invariants opératoires au cœur des schèmes qui sont pour le sujet les outils d'adaptation au réel. Ils permettent à ce dernier d'orienter et de guider son action. C'est la raison pour laquelle les analyses en didactique professionnelle s'attachent à identifier les invariants opératoires dans les activités des acteurs au travail (Pastré, 2011).

D'un point de vue méthodologique, la didactique professionnelle a connu quelques évolutions. Actuellement, c'est avant tout la recherche de jugements pragmatiques qui est mise en avant pour analyser l'activité, notamment dans le domaine de l'enseignement. Un jugement pragmatique représente "un énoncé tenu pour vrai qui permet de subsumer en les justifiant toute une série d'énoncés de circonstances » (p. 191).

Pour Vinatier analyser l'activité dans le champ de l'éducation, suppose en outre de tenir compte $d u$ fait qu'il s'agit d'une co-activité au cours de laquelle des enjeux interpersonnels - «place et rôle du sujet dans les échanges, son image, ce qui lui importe, ce qui le tracasse, ses motivations, ses valeurs» (2013, p.37) - peuvent parfois surdéterminer le contenu échangé.

L'auteur propose alors de distinguer deux types d'invariants en tension (Vinatier, 2012) :

- Les invariants situationnels : principes tenus pour vrais au regard de la tâche à accomplir ;

- Les invariants du sujet : principes éthiques, motivations personnelles.

\subsection{Les classes de situations}

Si l'activité enseignante est organisée, ce qui la rend analysable, se pose malgré tout, dans ce domaine professionnel particulier, la question d'identifier ce qui peut constituer des classes de situations. Il est difficile en effet dans l'enseignement de caractériser de façon précise des classes de situations même si des régularités sont observables (Vinatier, 2013). Il faut en fait les considérer comme des configurations de situations, c'est-à-dire des situations pour lesquelles « on a bien réussi à détacher des totalités singulières, des propriétés pertinentes, mais ce travail reste ouvert, inachevé et en devenir » (Pastré, 2011, pp. 243-244).

En EPS, Durand (2001), dans sa volonté de rendre compte de l'action des enseignants d'éducation physique, distingue deux phases caractéristiques dans le temps de la leçon: des phases de définition collective de l'action des élèves et des phases d'interaction de guidage plus individualisé. Une distinction similaire est reprise par Gal-Petitfaux (2011). Attachée à décrire les configurations d'activité collective qui structurent le déroulement de la leçon d'EPS, l'auteure a identifié trois grandes configurations : le début de la leçon (de l'arrivée des élèves à la première prise de parole de l'enseignant), les explications collectives (présentation collective des consignes au début de la leçon et de chaque situation d'apprentissage et bilans collectifs), la supervision de la pratique (ou gestion interactive, régulation, guidage, etc.).

Centrées sur le temps de la leçon, ces trois configurations ne rendent pas compte de l'activité mise en œuvre par les enseignants lorsqu'ils conçoivent leur projet 
d'enseignement. Or, il s'agit là selon nous d'une situation caractéristique de l'activité des enseignants qui témoigne, entre autres, de la façon dont les prescriptions curriculaires sont prises en compte, et à laquelle nous pouvons avoir accès à partir d'entretiens mais aussi à partir de l'analyse des traces objectives de l'activité professionnelle (Vinatier, 2013) considérées comme des indicateurs en actes de la production du curriculum (Brière-Guenoun, 2013).

\subsection{Objectifs de recherche}

31 Nous nous intéressons donc dans le cadre de cette recherche à la manière dont est mis en œuvre le curriculum effectif à partir de l'analyse in situ de l'activité d'étudiants-stagiaires en EPS, lors de leur stage en responsabilité. Plus précisément, nous cherchons à savoir ce qui oriente et guide leur activité dans des classes de situations caractéristiques de l'enseignement.

32 Pour cela, nous tentons d'identifier les organisateurs de cette activité, c'est-à-dire les invariants situationnels et les invariants du sujet ou, pour le dire autrement, les principes tenus pour vrais au regard de la tâche à accomplir et des enjeux interpersonnels. Nous souhaitons ainsi rendre compte de ce qui, en profondeur, oriente les choix opérés dans l'action, lorsque les étudiants-stagiaires sont aux prises avec le curriculum recommandé.

C'est à partir de la mise en évidence de ces logiques d'arrière-plan de l'action (Bucheton, 2009), que nous envisageons ensuite de décrire la façon dont ils s'approprient les recommandations, et donc de mettre en lumière leur rapport au curriculum recommandé, à l'injonction d'enseigner par compétences en recourant à des situations complexes notamment.

\section{Méthodologie}

\subsection{Participants}

Pour réaliser cette recherche, nous avons sollicité des étudiants en deuxième année de master MEEPS (métiers de l'enseignement en éducation physique et sportive) de la faculté des sports de Bordeaux. Au cours de l'année de formation, les étudiants sont en stage en responsabilité à $1 / 3$ temps deux jours par semaine. Ils préparent également les épreuves d'admission du certificat d'aptitude au professorat d'éducation physique et sportive (CAPEPS). Nous avons retenu, parmi les volontaires, quatre étudiants-stagiaires (4 garçons : Tom; Théo ; José ; Yan) enseignant le badminton au début de l'année scolaire : trois en collège et un en lycée.

\subsection{Recueil de données}

Vers la fin du premier trimestre, nous avons filmé et enregistré chaque étudiant lors d'une séance d'apprentissage. Les séances observées ont été les suivantes (voir tableau ciaprès).

Tableau 1 : Participants à l'étude et contexte (classe, séance et compétence attendue)

\begin{tabular}{|l|l|l|l} 
Nom & Classe & Séance & Compétence attendue des programmes
\end{tabular} 


\begin{tabular}{|l|l|l|l|}
\hline Tom & $5^{\mathrm{e}}$ & $\begin{array}{l}10^{\mathrm{e}} / 10 \\
\text { Théo }\end{array}$ & $\begin{array}{l}\text { Niveau } 1: \text { En simple, rechercher le gain loyal d'une rencontre en } \\
\text { choisissant entre renvoi sécuritaire et recherche de rupture sur des } \\
\text { volants favorables par l'utilisation de frappes variées en longueur ou en } \\
\text { largeur. Assurer le comptage des points et remplir une feuille } \\
\text { d'observation. }\end{array}$ \\
\hline José & $4^{\mathrm{e}} / 10$ & $\begin{array}{l}\text { Niveau } 2: \text { Rechercher le gain d'une rencontre en construisant le point } \\
\text { dès la mise en jeu du volant et en jouant intentionnellement sur la } \\
\text { continuité ou la rupture par l'utilisation de coups et trajectoires variés. } \\
\text { Gérer collectivement un tournoi et aider un partenaire à prendre en } \\
\text { compte son jeu pour gagner la rencontre. }\end{array}$ \\
\hline Yan & $1^{\mathrm{e}}$ & $\begin{array}{l}5^{\mathrm{e}} / 10 \\
8^{\mathrm{e}} / 10\end{array}$ & $\begin{array}{l}\text { Niveau 2: Rechercher le gain d'une rencontre en construisant le point } \\
\text { dès la mise en jeu du volant et en jouant intentionnellement sur la } \\
\text { continuité ou la rupture par l'utilisation de coups et trajectoires vairés. } \\
\text { Gérer collectivement un tournoi et aider un partenaire à prendre en } \\
\text { compte son jeu pour gagner la rencontre. }\end{array}$ \\
\hline $\begin{array}{l}\text { Niveau } 3: \text { Pour gagner le match, s'investir et produire volontairement } \\
\text { destrajectoires variées en identifiant et utilisant les espaces libres pour } \\
\text { mettre son adversaire en situation défavorable. }\end{array}$ \\
\hline
\end{tabular}

Avant chaque séance, nous avons réalisé un entretien ante (EA) dont l'objectif était que l'étudiant nous présente son projet d'enseignement en le justifiant. Les questions étaient les suivantes :

- Dans cette séance, qu'est-ce que tu veux apprendre aux élèves ? Pourquoi ?

- Dans quel dispositif? Pourquoi?

Nous avons ensuite réalisé avec chacun d'entre eux un entretien d'auto-confrontation simple (EAS). Pour ce faire, nous avons tout d'abord retranscrit l'ensemble des communications verbales des étudiants-stagiaires à partir des enregistrements vidéo et audio des séances observées. Afin de pouvoir mieux rendre compte du curriculum «tel qu'il se fait » - ce qui est enseigné et de quelle façon notamment -, nous avons identifié les moments pendant lesquels l'activité de l'étudiant est focalisée sur la transmission d'objets d'enseignement. Nous avons alors réduit la vidéo en conservant les extraits les plus significatifs présentés dans leur ordre chronologique d'apparition. La durée de chaque vidéo support de l'entretien était environ de 20 minutes. Nous avons dans un troisième temps réalisé un entretien d'auto-confrontation croisée conduit collectivement (EAC).

Les protocoles d'entretien d'auto-confrontation ont été conduits selon les recommandations méthodologiques développées par Goigoux (2007). Lors de l'entretien d'auto-confrontation simple, les étudiants-stagiaires étaient invités à commenter leur activité. Ils devaient se focaliser sur ce qui leur semblait important en essayant de centrer leurs commentaires sur les éléments invariants de leur activité. L'idée était qu'ils expliquent en quoi le déroulement des actions observées était habituel et dans quelle mesure il était similaire à celui qu'ils réalisent dans des situations comparables.

Lors de l'entretien d'auto-confrontation croisée (EAC), chaque étudiant devait commenter à tour de rôle l'activité vidéoscopée d'un autre étudiant du groupe. Les commentaires devaient préciser en quoi l'activité observée faisait écho, par similitude ou par contraste à ses propres manières de faire, à ses interrogations, à ses convictions ou à ses valeurs. Tout 
au long du visionnage commenté de la séance, les étudiants-stagiaires pouvaient s'exprimer et débattre. Un tel cadre dialogique, propice au développement de controverses professionnelles, donnait l'occasion de revenir sur ses propres expériences et de formuler les principes ou jugements pragmatiques et subjectifs qui orientent et guident les actions.

\subsection{Analyse des données}

\section{entretiens d'auto-confrontation simple (EAS). Pour ce faire, nous avons présenté} l'ensemble des verbatim dans un tableau à deux volets :

- Dans la colonne de gauche figurent les retranscriptions des communications et de l'entretien d'auto-confrontation

- Dans la colonne de droite figurent les résultats du premier temps de l'analyse

Cette analyse a consisté à répondre aux questions suivantes :

- De quelle classe de situations s'agit-il?

- Quelles sont les règles d'action mises en œuvre (RA)?

- En vue de quel(s) but(s) ?

- Quels sont les organisateurs de l'action, c'est-à-dire les énoncés ou principes tenus pour vrais ? Relèvent-ils de la situation ou du sujet?

- Quels sont les dispositifs proposés et les objets enseignés ?

L'identification des énoncés tenus pour vrais s'est faite en repérant, parmi tous les énoncés recueillis, le jugement suffisamment général permettant de fonder les autres. Lorsqu'il est arrivé que celui ne soit pas formulé par l'étudiant, nous avons nous-mêmes énoncé le jugement en procédant par inférence. Pour ne pas commettre d'erreur, Pastré (2011, p. 192) recommande de "s'assurer que les inférences qu'on peut tirer de ce jugement couvrent l'ensemble des autres énoncés ». Le tableau ci-dessous présente un extrait d'analyse d'un début de séance.

Tableau 2 : Extrait d'analyse du verbatim des communications et de l'entretien d'auto-confrontation simple

\begin{tabular}{|c|c|}
\hline Verbatim & Analyse \\
\hline $\begin{array}{l}\text { Communications } \\
\text { La semaine dernière, première séance, qu'est-ce qu'on } \\
\text { avait dit? Ce que j'avais vu moi surtout c'est que les } \\
\text { échanges, c'était justement des échanges, on n'était pas } \\
\text { vraiment dans la rupture, et le service, y'avait un gros } \\
\text { souci au niveau du service. Déjà, y'en a qui connaissaient } \\
\text { pas comment on sert règlementairement. }\end{array}$ & $\begin{array}{l}\text { Situation: Présentation séance } \\
\text { RA: revenir sur ce qui a été fait la } \\
\text { semaine précédente } \\
\text { RA: justifier le thème de la séance au } \\
\text { regard des conduites des élèves } \\
\text { But: faire comprendre aux élèves l'intérêt } \\
\text { du thème de la séance } \\
\text { Objet d'enseignement: le service }\end{array}$ \\
\hline $\begin{array}{l}\text { Entretien d'auto-confrontation (EAS): } \\
\text { Là, je leur explique le travail sur lequel on allait } \\
\text { partir, que j'avais prévu et je fais le lien avec ce que } \\
\text { j'avais vu chez eux la semaine dernière pour qu'ils } \\
\text { comprennent pourquoi on va partir sur ça. }\end{array}$ & $\begin{array}{l}\text { Invariant situationnel : faire le lien entre } \\
\text { ce qui va être fait et ce qui a été fait la } \\
\text { semaine précédente permet aux élèves de } \\
\text { leur faire comprendre l'intérêt du thème } \\
\text { de la séance, pourquoi ils vont faire telle } \\
\text { ou telle chose }\end{array}$ \\
\hline
\end{tabular}


En nous appuyant sur un travail de comparaison systématique des buts, des règles d'action et des invariants, nous avons été amené à redéfinir et surtout à réduire le nombre de classes de situations. Nous avons finalement distingué trois grandes classes de situations comprenant pour certaines d'entre elles des sous-classes :

- Les situations relatives aux activités de conception : conception du cycle, conception de la séance, conception des situations

- Les situations relatives aux activités d'explication collective: explication des consignes (séance et situation d'apprentissage) et bilan (situation et séance)

- Les situations relatives aux activités de régulation

Une fois ce travail réalisé, nous avons construit un document de synthèse pour chaque étudiant présentant, dans chaque classe de situations identifiée, les buts, les règles d'action, les invariants, les dispositifs et les objets d'enseignement.

Nous avons alors entrepris un travail de comparaison entre les étudiants-stagiaires. Pour chaque classe de situations, nous avons relevé les points communs et les divergences en nous centrant plus particulièrement sur les principes tenus pour vrais. Cela nous a amené parfois à reformuler certains principes partagés.

L'analyse des entretiens ante séance et d'auto-confrontation croisée conduit collectivement s'est faite selon les mêmes principes. Elle nous a permis, selon le principe de la triangulation des données, de valider, d'affiner et de compléter les résultats obtenus. Nous avons à partir de là procédé à la mise en forme finale de la présentation des résultats.

\section{Résultats}

À l'issue de ce travail d'analyse, nous avons mis en évidence une quarantaine de principes, partagés (ou non) selon les cas. Certains sont des invariants situationnels, d'autres des invariants du sujet. Ces principes sont les organisateurs des trois grandes classes de situations.

Certains principes occupent une place de choix, dans la mesure où ils sont tenus pour vrais par tous les étudiants-stagiaires, mais aussi et surtout, parce qu'ils paraissent fonder en grande partie tous les autres. Nous avons dès lors considéré qu'il s'agissait là de " grands principes partagés ». Leur particularité est de ne pas être rattachés à une classe de situations. Peu circonstanciés, ils ont une portée générale. Ce sont eux qui vraisemblablement orientent prioritairement l'activité des étudiants-stagiaires lorsqu'ils interviennent dans leur classe.

Ne pouvant, dans le cadre de cet article, présenter l'ensemble des principes tenus pour vrais par les étudiants, nous rendrons compte prioritairement de ceux qui nous renseignent sur le rapport des étudiants au «curriculum recommandé». Ce sont majoritairement ceux qui organisent les activités de conception.

Avant cela toutefois, et pour contextualiser ces dernières, il nous paraît important de revenir sur les "grands principes partagés " qui constituent selon nous une sorte d'arrière-plan profond influençant les choix opérés dans l'action. 


\subsection{Les « grands principes partagés » qui organisent l'intervention des étudiants dans leur classe}

51 Pour l'ensemble des quatre étudiants, ce qui organise leur activité, lorsqu'ils conduisent la classe, se situe du côté de leur posture d'enseignant et de la relation avec les élèves, et renvoie à des questions identitaires. Pour chacun d'entre eux en effet, il importe avant tout de ne pas "être le chef», de ne pas instaurer un rapport magistral : l'élève doit se sentir pris en charge et reconnu personnellement.

On pourrait résumer de la façon suivante les principes qui guident l'activité des étudiants :

- Il faut essayer d'être proche des élèves, de ne pas un instaurer un rapport autoritaire et magistral mais un rapport amical.

- Il faut s'intéresser aux élèves, être en relation avec chacun d'entre eux, avoir des échanges ou même de réelles discussions.

53 C'est à l'occasion de la façon dont ils décrivent leur position au moment de la présentation des consignes, que cette préoccupation est la plus vivace :

- Yan « C'est une position que je n'aime pas être debout face aux élèves

- Tom «Ça fait supérieur, ça fait un peu : “je suis le chef” » (EAC).

54 Une telle relation cependant ne doit pas dépasser certaines limites. Il importe en effet, même si le rapport hiérarchique est difficile à assumer parfois qu'il n'y ait pas de confusion de statut. C'est pourquoi cette relation professeur/élève, aussi proche soit-elle, ne doit pas se transformer en une relation entre amis : "Si je les laisse vraiment s'asseoir à côté de moi, ça fait vraiment copains/copains et là je pense que eux dans leur tête, ils n'ont plus l'image: "C'est quand même le prof même s'il est très proche de nous, [...] qu'il fait pas le maître qui dicte " (Tom/EAC).

Un autre "grand principe organisateur» de l'activité pourrait par conséquent être formulé :

- Il faut, tout en étant proche des élèves, renvoyer une image de professeur afin qu'il n'y ait pas de confusion de statut.

Plus généralement, ce qu'expriment les étudiants à travers cette quête de proximité et d'échanges avec les élèves, c'est selon nous, un besoin d'être à la fois reconnu - "C'est quand même très important d'avoir des connaissances, de montrer aux élèves qu'on maitrise. On est le référent quand même " (Yan/EAS) -, mais aussi apprécié des élèves : "Il faut que tu arrives à te faire quand même, bien aimé de tes élèves. " (José/EAC).

Ces éléments nous amènent dès lors à formuler un autre « grand principe » organisateur :

- Il faut être reconnu et apprécié des élèves.

Les derniers grands principes partagés que nous avons relevés ont comme spécificité d'influencer l'activité de régulation dont ils sont un organisateur puissant :

- Il faut aller voir tous les élèves, s'occuper de chacun d'entre eux, donner des conseils à tout le monde pour qu'ils sentent qu'on s'occupe d'eux, pour leur montrer qu'on est là. 
donner des conseils à tous les élèves, être attentif à chacun, va de pair avec la manière de s'adresser aux élèves. Il ne suffit pas en effet d'aller voir tous les élèves, il faut aussi instaurer avec chacun d'entre eux un mode de relation adéquat :

- Il est important d'être positif, d'encourager les élèves, de les féliciter, les rassurer, plaisanter avec eux, les mettre en confiance, les valoriser, pour les maintenir dans l'activité et favoriser leur engagement.

\subsection{Les principes qui organisent les activités de conception}

\subsubsection{Les principes prioritaires}

$60 \mathrm{Au}$ sein de cette classe de situations, il est possible de distinguer des principes différents, selon que les activités de conception concernent l'organisation du cycle, des séances ou des situations d'enseignement/apprentissage. Certains d'entre eux cependant semblent prioritaires par rapport à d'autres et ont vraisemblablement une portée plus générale. C'est le cas notamment du principe selon lequel :

- Pour que les élèves s'engagent dans l'activité, pour qu'ils s'impliquent et progressent, il faut qu'ils prennent du plaisir, qu'ils s'amusent et qu'il y ait un bon climat de classe.

61 Il s'avère ainsi que pour les quatre étudiants le plaisir des élèves est une condition nécessaire voire indispensable à leur engagement. Il est important par conséquent pour eux de choisir des situations qui procurent avant tout du plaisir et de l'amusement: " C'est ludique. Je suis sûr qu'ils vont se mettre dedans, qu'ils vont rigoler, qu'ils vont accrocher au truc. » (Yan/EAC).

Tous s'efforcent alors de proposer des situations ludiques, c'est-à-dire des situations non répétitives, sous formes de défi, ou présentant de l'incertitude : «Je trouve que c'est bien, ils ont l'air d'être tous pris au jeu et comme il y a une petite incertitude sur quel côté on va partir, c'est un peu la compétition entre tous. » (Théo/ EAC)

63 Au principe précédent s'articule ainsi le principe suivant :

- Les situations proposées aux élèves doivent être ludiques et amusantes pour les élèves, et ainsi susciter leur intérêt.

\subsubsection{Les principes qui organisent les activités de conception du cycle et de la séance}

Hormis José qui s'appuie sur des conduites typiques à partir desquelles il constitue des groupes et fixe des objectifs différenciés (il se réfère en cela à ce que lui a suggéré son tuteur), les trois autres étudiants-stagiaires procèdent de la même façon pour démarrer et organiser leur cycle. Ils s'appuient à la fois sur la compétence attendue et sur l'évaluation diagnostique réalisée en début de cycle pour déterminer les objets d'enseignement prioritaires.

- «J'ai découpé la compétence attendue, je me la suis bien relue, les fiches ressources surtout. Je me base sur ça au départ, parce que sinon on ne sait pas sur quoi partir. [...] Je découpe, je vois globalement les différents éléments et je me dis va falloir bosser tout ça. » (Tom/EA) 
- «L'idée c'est de travailler tous les aspects de la compétence attendue et qu'ils puissent tout mobiliser ensemble.» (Théo/EA)

Pour les étudiants cependant, le match ou la situation globale ne sont pas qu'un " dessert » (Yan) pour l'élève, c'est aussi l'occasion pour eux d'apprendre et de réinvestir ce qu'ils ont vu auparavant dans la séance : « Moi je sais que je ne fonctionne pas souvent sur des matchs où c'est que des matchs, [...] c'est un match à thème, un match à bonus, c'est pas une opposition pour une opposition. C'est quand même une opposition pour apprendre quelque chose ou bien remettre en place ce qu'on a essayé d'apprendre pendant la séance » (Tom/EAC). On retrouve en 
fait ici l'idée de réinvestissement mais dont on peut considérer qu'elle s'applique plus particulièrement à la situation de match :

- Prévoir un match ou une situation globale aménagée en fin de séance permet aux élèves de réinvestir ce qu'ils ont vu tout au long de la séance.

\subsubsection{Les principes qui organisent les activités de conception des situations d'apprentissage}

71 Les situations choisies par les étudiants sont bien sûr très différentes entre elles dans la mesure où elles sont ciblées sur des objets d'enseignement différents. Un point commun ressort cependant : tous les étudiants proposent très souvent des situations avec points bonus. Celles-ci sont en effet, selon eux, particulièrement intéressantes: "J'aime bien parce que je trouve que ça lie à la fois cet objectif de match qui guide (motive les élèves) tout en étant très éducatif [...] ça donne un aménagement du milieu qui pour moi les oblige à mettre en place ce sur quoi on a travaillé » (Tom/EAS).

72 Apparaît ici un nouveau principe, en lien avec les précédents, qui pourrait s'énoncer ainsi :

- Il est intéressant de proposer des situations avec point bonus car elles répondent au besoin de jeu des élèves et leur permettent de viser l'objectif de la séance. Elles obligent les élèves à réinvestir ce qu'ils ont travaillé précédemment.

73 Si les quatre étudiants-stagiaires partagent ce point de vue, ils ne sont pas tout à fait d'accord en revanche sur la manière de faire vivre ces situations aux élèves. Pour certains d'entre eux, recourir aux points bonus n'est intéressant que si les élèves comprennent l'intérêt de marquer de tels points, ce qui suppose alors d'utiliser des fiches d'observation.

Se pose ici, pour les étudiants-stagiaires observés, la question de savoir s'il faut proposer ou pas des situations confrontant les élèves à des rôles sociaux différents: celui d'observateur notamment. Bien que tous soient d'accord sur le principe, on ne peut pas dire pour autant que celui-ci oriente l'activité de tous les étudiants. L'un d'entre eux exprime sa réserve de la façon suivante: "Je pense que c'est un peu de l'utopie de leur faire faire ça. Je préfère qu'ils soient dans le jeu et qu'ils comptent [...] plutôt que vraiment tenir une feuille de stat: j'ai fait huit amortis, trois dégagés »(Tom/EAS). Ce qui est mis en avant surtout, c'est le fait que les élèves n'adhèrent pas à ce type de travail, et qu'il vaut mieux, en tant que professeur débutant, se centrer sur l'aspect moteur. Ils remettent à plus tard ce que d'autres s'efforcent de mettre en place mais avec difficulté toutefois : "Je pense que c'est vraiment primordial de se rendre compte que ce que l'on a travaillé en mettant les points bonus, c'est ce qui permet de gagner les points, et qu'ils le voient sur le papier [...]. Il faut les amener à réfléchir sur cette fiche et ça je trouve ça difficile ». (Théo/EAC)

Finalement, deux principes contradictoires, semblent pouvoir être énoncés :

- Les situations d'observation, même si elles sont intéressantes, ennuient les élèves. Il vaut mieux privilégier le jeu et l'activité motrice.

- Il est intéressant de faire vivre différents rôles sociaux aux élèves qui permettent aux élèves de comprendre le sens des apprentissages.

Deux autres points peuvent être mis en avant concernant les tâches de conception des situations d'apprentissage. Le premier renvoie à la question de la différenciation pédagogique. Alors que pour José il est judicieux de proposer des situations différenciées, 
pour les autres en revanche, cela est difficilement recevable. Le principe qui organise leur activité se fonde en grande partie sur l'idée qu'ils se font de leur mission :

- Un enseignant doit apprendre la même chose à tout le monde même si c'est à des degrés différents. C'est la raison pour laquelle, il ne doit pas proposer de situations différentes aux élèves hormis quelques aménagements et consignes différenciés.

Le dernier point a lui aussi fait l'objet d'un débat entre les étudiants lors des entretiens d'auto-confrontation croisée. Il a porté cette fois sur la pertinence des situations analytiques ou décontextualisées.

Pour les uns :

- Il est intéressant de proposer des situations décontextualisées pour permettre aux élèves de construire des connaissances susceptibles d'être réinvesties dans des situations plus globales.

Pour l'un d'entre eux :

- Les situations d'apprentissage favorables aux progrès des élèves sont celles qui les confrontent à des problèmes proches de la logique du jeu.

\section{Discussion}

Les résultats mettent en évidence trois préoccupations principales chez les étudiantsstagiaires observés lorsqu'ils sont aux prises avec le curriculum recommandé : construire sa posture d'enseignant; susciter et maintenir les élèves dans l'activité ; faire apprendre les élèves. Ces préoccupations sont à voir, selon nous, comme les principaux soubassements qui orientent leur activité, comme ses organisateurs pragmatiques dominants (Pastré, Mayen \& Vergnaud, 2006). Différentes de celles mises en évidence par Bucheton et Soulé (2009), elles présentent néanmoins des caractéristiques similaires: elles agissent simultanément de façon dynamique (elles sont plus ou moins présentes selon le moment de la leçon) mais surtout elles sont hiérarchisées. Cependant, alors que l'étayage est considéré par Bucheton et Soulé $(2009$, p. 36) comme un organisateur central de l'agir enseignant, « un concept central, hiérarchiquement supérieur aux autres », il est ici, si on l'assimile au « faire apprendre », un organisateur inférieur aux deux autres.

81 Nous présentons ci-après ces trois préoccupations hiérarchisées au fondement de l'activité des étudiants-stagiaires lors de leurs premières expériences d'enseignement.

\subsection{Construire sa posture d'enseignant}

82 Nous l'avons vu lors de la présentation des grands principes partagés, ce qui préoccupe prioritairement les étudiants-stagiaires en EPS lors de leur premier stage en responsabilité, c'est de trouver leur place auprès des élèves : ni ami, ni «chef », cette place n'est pas évidente à trouver. Résultat du passage du statut d'enseigné à celui d'enseignant, il est probable qu'elle évolue au fil de l'année.

Pour autant, contrairement à ce qui a pu être mis en avant dans d'autres contextes (Goigoux et al., 2009; Vinatier, 2012), ce n'est pas tant le fait d'avoir de l'autorité sur le groupe qui prime que celui d'être proche des élèves. Cela ne signifie pas néanmoins qu'il faille conclure à l'absence d'une telle préoccupation chez les enseignants débutants en EPS. Peut-être malgré tout peut-on suggérer que pour les étudiants-stagiaires d'EPS, les 
difficultés d'entrée dans le métier seraient moins importantes que pour d'autres. Ces résultats viendraient du coup confirmer ceux mis en évidence par ailleurs (Roux-Pérez, 2012).

Ainsi, devant une épreuve moins difficile à surmonter, les étudiants-stagiaires seraient davantage focalisés sur le fait d'entretenir avec leurs élèves des relations amicales propices à un climat de classe agréable. Moins préoccupés par la gestion et le contrôle de la classe, n'ayant pas l'impression « d'être mis dans l'arène », ils privilégient le registre de la relation. L'image qu'ils veulent renvoyer et avoir d'eux-mêmes est celle d'un professeur qui s'intéresse aux élèves, qui les reconnaît en tant que personne et qui les respecte. C'est de cette façon qu'ils pourront alors être reconnus et appréciés.

Se construire en tant qu'enseignant, c'est enfin pour les étudiants-stagiaires « endosser » ou "revêtir » leur rôle : celui d'aider les élèves à construire des compétences et ainsi justifier leur place au sein de l'établissement et, plus généralement, dans le métier. Ils sont auprès des élèves pour enseigner et non pour les « faire bouger ». Cette préoccupation s'exprime au moment des bilans notamment - sur lesquels nous n'avons pu revenir dans le cadre de cet article - lorsque les étudiants reviennent sur ce qui a été appris. Sans doute s'agit-il pour eux d'institutionnaliser les comportements adéquats, mais c'est surtout et avant tout, parce qu'en agissant ainsi ils se « sentent » enseignant et peuvent se reconnaître et être reconnus en tant que tel.

\subsection{Susciter et maintenir l'engagement des élèves}

Sur fond de ces premières préoccupations renvoyant à des idéaux et des enjeux intersubjectifs, les étudiants-stagiaires s'efforcent aussi de susciter l'engagement des élèves et de les maintenir en activité. Ce sont ici l'enrôlement et l'implication des élèves dans les tâches proposées qui les préoccupent (Goigoux et al., 2009). Ils recourent pour cela à différentes stratégies dont la plupart d'entre elles sont partagées.

On l'a vu, il s'agit avant tout de proposer aux élèves des situations ludiques pour qu'ils prennent du plaisir et qu'ils s'amusent. Une telle préoccupation peu mise en avant jusqu'à présent chez les enseignants débutants, tient peut-être à la spécificité de la discipline, au fait qu'elle s'appuie sur le jeu, l'action, le mouvement et qu'elle soit largement plébiscitée par les élèves (Cosnefroy, 2007). Les élèves venant, pour la majorité d'entre eux avec plaisir, les enseignants profiteraient en quelque sorte de ce capital de départ à entretenir pour favoriser l'engagement des élèves. La place dévolue au match ou le temps accordé aux moments de jeu s'inscrivent sans doute dans cette même logique.

Le maintien des élèves dans l'activité passe aussi, pour les étudiants-stagiaires, par l'intérêt qui leur est apporté et la valeur qui leur est accordée. Les élèves doivent donc sentir que le professeur leur porte de l'attention, qu'il est présent, bienveillant et qu'il s'occupe d'eux. C'est la raison principale pour laquelle ils circulent dans la classe et passent voir tous les élèves. S'ils donnent des conseils à chacun d'entre eux, c'est certes pour les aider à réussir la tâche, mais c'est aussi et surtout pour les encourager à poursuivre, pour éviter qu'ils ne décrochent. Ils ne cessent dès lors d'encourager, de valoriser, de féliciter, et ce surtout, lorsqu'ils s'adressent à des élèves en difficulté. Dans de tels cas, ils minimisent les erreurs, ils rassurent, bref, ils font tout pour que ces élèves persévèrent. Se sentant eux-mêmes souvent impuissants face aux difficultés de ces élèves, ils essaient malgré tout de surmonter leur propre découragement. 


\subsection{Faire apprendre les élèves}

89 La troisième préoccupation - mettre les élèves en "activité » constructive - est censée apparaitre assez tardivement chez les débutants sauf lorsque les situations sont moins difficiles (Goigoux et al., 2009; Saujat, 2005). Force est de constater que pour les étudiants-stagiaires observés, elle est bien présente. Certes, il se trouve qu'aucun d'entre eux n'avait affaire à des élèves difficiles. On peut dès lors supposer qu'il aurait pu en être autrement dans des contextes différents. Il n'en reste pas moins que confrontés malgré tout au " choc de la réalité ", ils ne renoncent pas à leurs idéaux (Roux-Pérez, 2012) même s'ils redéfinissent en partie la tâche prescrite (Daguzon, 2009) et reportent à plus tard ce qu'ils estiment être pour l'instant prématuré.

90 Ainsi, au-delà des différentes stratégies mises en œuvre, tous s'efforcent de rendre les élèves plus compétents et de construire des contenus adaptés : jouer en profondeur et en largeur, varier et optimiser les coups, viser les espaces libres, mettre l'adversaire en difficulté dès la mise en jeu, tenir le rôle de coach et d'observateur, etc. Ils s'appuient majoritairement pour cela, on l'a vu, sur les programmes officiels et les fiches ressources, perçus non pas comme un cadre contraignant mais comme une aide au choix des objets d'enseignement : "J'ai découpé la compétence attendue [...]Je me base sur ça au départ, parce que sinon on ne sait pas sur quoi partir» (Tom, EA). Il est probable en outre que ces textes institutionnels aient également une influence sur les décisions prises lors de la conception des situations. Le recours massif aux situations avec points bonus par exemple n'est sans doute pas étranger au fait qu'elles soient préconisées pour l'évaluation au brevet (BO, 2012).

91 Mais l'intérêt porté aux apprentissages des élèves ne se limite pas aux choix des situations, il est présent également lorsque les étudiants interviennent auprès des élèves. Ainsi, même si nous n'avons pu le développer dans le cadre restreint de cet article, il s'avère que lors des activités d'explication collective et de régulation, démontrer, expliquer ce qu'il faut faire, justifier, faire comprendre, observer, donner des conseils, sont autant de règles d'action mises en œuvre pour faire apprendre les élèves et pour qu'ils s'impliquent dans les apprentissages. Par conséquent, nous retiendrons que si cette préoccupation n'est pas première, elle est de toute évidence réelle. Et l'on ne pourrait comprendre l'activité de ces étudiants sans prendre en compte le fait qu'elle est aussi organisée autour des apprentissages des élèves.

\section{Conclusion : le rapport au curriculum recommandé}

92 Plusieurs conclusions sur le rapport au curriculum recommandé des étudiants-stagiaires d'EPS en formation par alternance, nous paraissent pouvoir être tirées de l'analyse de ces résultats.

Il s'avère tout d'abord que l'entrée relativement " douce » de ces étudiants dans le métier leur donne la possibilité d'enseigner ou de "faire » la classe (Saujat, 2004). Et même si comme la plupart des débutants, ils sont d'abord préoccupés par des enjeux relationnels (Vinatier, 2013) et identitaires (Roux-Perez, 2012) et la mise au travail des élèves (Guibert, Lazuech \& Rimbert, 2008), ils sont aussi soucieux de transmettre les savoirs à l'œuvre dans les compétences et donc de faire ce qui est attendu d'eux. Ainsi, tous sont investis d'une même mission: enseigner. Mais cela est insuffisant, il s'agit pour eux non pas 
seulement d'enseigner mais de faire réussir tous les élèves - bien qu'ils ne sachent pas trop comment faire - et ce, en apprenant la même chose à tout le monde même si l'un d'entre-eux (José) a procédé différemment suite aux conseils de son tuteur.

On constate ensuite, au-delà de ces valeurs partagées, que les étudiants procèdent à des « arrangements personnels» du curriculum recommandé. Celui-ci, on l'a vu, est une ressource dans la mesure où il oriente l'activité de conception notamment, mais il est surtout, selon nous, un cadre qui à la fois contient mais ne contraint pas. Il évite quelques errements tout en laissant des marges de manœuvre possibles.

Les étudiants-stagiaires observés retiennent ainsi de ce cadre ce qui peut répondre à leurs préoccupations principales. Ils privilégient par exemple les situations bonus parce qu'elles sont ludiques et motivantes pour les élèves, parce qu'elles ne sont pas trop difficiles à organiser, parce qu'elles sont ciblées sur un objet d'enseignement en lien avec la compétence attendue (par exemple, la variété des coups pour Tom, le jeu dans la latéralité pour José ou la rupture au service pour Théo), et enfin parce qu'elles leur permettent de « tenir » leur rôle.

Ils sont plus réticents en revanche à mettre en place des situations avec rôles sociaux privilégiant notamment les moments d'observation, d'échanges et de réflexion sur l'action. Il s'agit bien là pourtant de recommandations auxquelles ils accordent du crédit. Cependant, elles risqueraient de les mettre en danger. Et d'ailleurs certains de ceux qui ont essayé ont dû renoncer sous la pression des élèves. Mieux vaut par conséquent selon eux, pour ne pas perdre la face, se limiter à ce qui est recommandé mais accessible : faire arbitrer les élèves par exemple.

De la même manière, convaincus de l'intérêt de faire des allers-retours entre des situations globales et des situations plus ciblées sans ordre préétabli (injonction qui relève des prescriptions secondaires), les quatre étudiants-stagiaires n'envisagent pourtant pas d'aller à l'encontre de ce qui relève selon eux du genre des enseignants d'EPS : « ils ont [...] l'habitude, tous les ans ils font ça, c'est toujours des situations puis ça finit par un match. Ils le savent.» (Théo, EAC)

En s'arrangeant de la sorte avec le curriculum recommandé, les étudiants-stagiaires ne se détournent pas pour autant complètement de l'orthodoxie curriculaire (didactique et pédagogique) actuelle qui consiste à faire travailler sur des activités suffisamment globales, qui ont du sens et dont la fonctionnalité apparaît à l'élève (Rey et al., 2006). Ils ne cessent en effet de justifier les apprentissages en s'appuyant sur les conduites initiales des élèves et en mettant en avant leur utilité dans le match. Ils recourent par ailleurs très souvent à des situations plus ou moins complexes nécessitant de mobiliser les acquis et de combiner un ensemble de ressources.

99 Certes, on l'a vu, il leur est difficile d'innover et ils reproduisent la routine professionnelle dénoncée par Falco (2013): des situations d'apprentissages qui se succèdent conduisant vers une situation plus globale en fin de séance dans laquelle l'élève doit faire la synthèse de ce qu'il a vu auparavant. Pour autant, il serait hasardeux de dire qu'il s'agit là exclusivement d'un modèle cumulatif par emboîtement de situations consistant à ne laisser découvrir l'exigence d'avoir à mobiliser à bon escient les ressources qu'au moment du contrôle de fin de cycle (Ibid.). Il s'agirait bien dès lors d'un enseignement relevant plutôt d'une logique d'intégration (Rogiers, 2010), accordant peu de temps aux moments de réflexion sur l'action mais favorisant toutefois le développement de compétences chez les élèves. 
De tels constats invitent à interroger la formation pour chercher à l'optimiser. L'analyse de l'activité dans le cadre de la didactique professionnelle a en effet en vue le développement des compétences professionnelles (Pastré, Mayen \& Vergnaud, 2006). Or, on vient de le voir, les étudiants-stagiaires ont tendance à simplifier les situations dans lesquelles ils se trouvent et à remettre à plus tard la mise en place de dispositifs didactiques innovants plus difficiles à gérer. Par conséquent, si l'on considère que l'émancipation des élèves suppose qu'ils soient confrontés à des situations complexes, sollicitant de leur part une démarche réflexive, alors il faut envisager, comme le suggère Vinatier (2013), des modalités de formation qui donnent aux étudiants-stagiaires la possibilité d'expérimenter, dans un cadre sécurisé, des situations à risques plus difficiles à conduire. Ce qui suppose alors d'aller à l'encontre d'une formation qui expose les étudiants à une gradation des difficultés auxquelles ils peuvent se trouver confrontés (Ibid.) et de donner toute sa place aux enjeux épistémiques des séances.

\section{BIBLIOGRAPHIE}

Baillauquès, S., \& Breuse, E. (1993). La première classe. Paris : ESE.

Brière-Guenoun, F. (2013). Physical Education Curricula in France. How to engage teachers in change? Oral presentation at the European Seminar on "Curriculum Development in Physical Education and Health across Europe". Utrecht University, June 20 and 21, 2013.

Bucheton, D. (2009). Le modèle de l'agir enseignant et ses ajustements. In D. Bucheton (Dir.) L'agir enseignant : des gestes professionnels ajustés (pp. 25-68). Toulouse : Octarès.

Bucheton, D., \& Soulé, Y. (2009). Les gestes professionnels et le jeu des postures de l'enseignant dans la classe : un multi-agenda de préoccupations enchâssées. Éducation et didactique, 3, 29-48.

Butlen, D., Charles-Pézard, M. \& Masselot, P. (2009). Gestes et routines professionnels : un enjeu pour analyser et intervenir sur les pratiques enseignantes. Actes Colloque international Espace Mathématique Francophone, Dakar, 6-10 avril 2009.

Chaliès, S., \& Durand, M. (2000). Note de synthèse : l'utilité discutée du tutorat en formation initiale des enseignants. Recherche et formation, 35, 145-180.

Crahay, M. (2006). Dangers, incertitudes et incomplétude de la logique de la compétence en éducation. Revue française de pédagogie, 154, 97-110.

Crahay, M., Audigier, F., \& Dolz, J. (2006). En quoi les curriculums peuvent-ils être objet d'investigation scientifque ? In F. Audigier, M. Crahay \& J. Dolz (Dir.), Curriculum, enseignement et pilotage (pp. 7-37). Bruxelles : De Boeck Université.

Cosnefroy, L. (2007). Le sentiment de compétence, un déterminant essentiel de l'intérêt pour les disciplines scolaires. L'orientation scolaire et professionnelle, 36(3), mis en ligne le 15 septembre 2012, consulté le 01 juin 2012.

Daguzon, M. (2009). De la prescription à la redéfinition de la tâche d'enseignement (étude longitudinale). In R. Goigoux, L. Ria, \& M.C. Toczek-Capelle (Dir.), Les parcours de formation des enseignants débutants (pp. 101-120). Clermont-Ferrand : Presses Universitaires Blaise Pascal. 
Daguzon, M., \& Goigoux, R. (2012). Apprendre à faire classe. Les apprentissages professionnels des professeurs des écoles en formation par alternance. Revue française de pédagogie, 181, 27-42.

Durand, M. (2001). Chronomètre et survêtement. Reflets de l'expérience quotidienne d'enseignants d'éducation physique. Paris : Revue EPS.

Falco, A. (2013). Situations complexes en EPS : la nécessité d'accompagner la conception et les mises en œuvre d'un enseignement par compétences. Enseigner l'EPS, Revue de l'AE-EPS, 261, 21-28.

Forquin, J.C. (2008). Sociologie du curriculum. Rennes : PUR, collection Paideia.

Gal-Petitfaux, N. (2011). La leçon d'éducation physique et sportive : formes de travail scolaire, expérience et configurations d'activité collective dans la classe. Note de synthèse en vue de l'obtention d'une Habilitation à diriger des recherches. Université Blaise Pascal de Clermont-Ferrand2.

Goigoux, R. (2007). Un modèle d'analyse de l'activité des enseignants. Education et didactique, 1(3), 47-69.

Goigoux, R., Ria, L., \& Toczek-Capelle, M.C. (2009). Les parcours de formation des enseignants débutants. Clermont-Ferrand : Presses Universitaires Blaise Pascal.

Goodson, I. F. (2008). Curriculum reform and curriculum theory: A case of historial amnesia. In N. Nigel (Ed.). Curriculum and the Teacher. 35 years of the Cambridge Journal of Education. London : Routledge.

Guibert, P., Lazuech, G., \& Rimbert, F. (2008). Enseignants débutants, "faire ses classes ». L'insertion professionnelle des professeurs du second degré. Rennes : Presses universitaires de Rennes.

Hirtt, N. (2009). L'approche par compétences : une mystification pédagogique. L'école démocratique , 39, 1-34.

Legendre, M.-F. (2008). La notion de compétence au cœur des réformes curriculaires : Effet de mode ou moteur de changements en profondeur? In F. Audigier \& N. Tutiaux-Guillon (Dir.), Compétences et contenus : les curriculums en questions (pp. 27-50). Bruxelles : De Boeck.

Lenoir, Y. (2006). Du curriculum formel au curriculum enseigné : comment des enseignants de primaire comprennent et mettent en œuvre le nouveau curriculum de l'enseignement primaire. In M. Crahay, F. Audigier \& J. Dolz (Dir.), J. Curriculum, enseignement et pilotage (pp. 119-141). Bruxelles : De Boeck.

Lenzen, B. (2012). Les activités curriculaires des enseignants d'EPS, entre prescriptions et liberté : une revue de littérature. eJRIEPS, 27, 27-65.

Martinand, J.L. (2001). Matrices disciplinaires et matrices curriculaires : le cas de l'éducation technologique en France. In C. Carpentier (Ed.), Contenus d'enseignement dans un monde en mutation : permanences et ruptures (pp. 249-269). Paris : L'harmattan.

Musard, M., Robin, J.F. \& Caty, D. (2009). La construction des cycles d'acrosport : une démarche originale des enseignants d'EPS. eJRIEPS, 16, 73-85.

Numa-Bocage, L., Clauzard, P. \& Pastré, P. (2012). Activité enseignante et didactique professionnelle : analyse de la co-activité en situation scolaire. In M. Altet, M. Bru \& C. Blanchard-Laville (Coord.), Observer les pratiques enseignantes (pp. 207-221). Paris : L'Harmattan.

Pasco, D., \& Ennis, C. (2009). Les orientations de valeur des enseignants. Une revue de question en éducation physique. Movement \& Sport Sciences, 66, 85-112.

Pastré, P., Mayen, P., \& Vergnaud, G. (2006). Note de synthèse : La didactique professionnelle. Revue française de pédagogie, 154, 145-198. 
Pastré, P. (2011). La didactique professionnelle : approche anthropologique du développement chez les adultes. Paris : Presses universitaires de France.

Perrenoud, P. (1993). Curriculum : le formel, le réel et le caché. In J. Houssaye (Ed.), La pédagogie : Une encyclopédie pour aujourd'hui (pp. 61-76). Paris : ESF.

Poggi, M.P., Verscheure, I., Musard, M., \& Lenzen, B. (2010). Vers une approche socio-didactique en intervention. In M. Musard, M. Loquet et G. Carlier (Dir.), Sciences de l'intervention en EPS et en sport. Résultats de recherche et fondements théoriques (pp. 159-178). Paris : Revue EPS.

Rey, B., Carette, V., Defrance, A., \& Kahn, S. (2006). Les compétences à l'école. Apprentissage et évaluation. Bruxelles : De Boeck.

Rey, B. (2014). La notion de compétence en éducation et formation. Enjeux et problèmes. Bruxelles : De Boeck.

Rey, O. (2010). Contenus et programmes scolaires : comment lire les réformes curriculaires? Dossier d'actualité de la VST, 53. En ligne http://www.inrp.fr/vst/LettreVST/53-avril-2010.php

Ria, L. (2009). De l'analyse de l'activité des enseignants débutants en milieu difficile à la conception de dispositifs de formation. In M. Durand \&t L. Filliettaz (Eds.), Travail et Formation des Adultes (pp. 217-243). Paris : PUF.

Ria, L., \& Rouve, M.E. (2009). Observatoire du développement professionnel des néo-titulaires en collèges « ambition réussite ». In R. Goigoux, L. Ria \& M.C. Toczek-Capelle (Dir.), Les parcours de formation des enseignants débutants (pp. 255-269). Clermont-Ferrand : Presses Universitaires Blaise Pascal.

Robert, A. (2008). La double approche didactique et ergonomique pour l'analyse des pratiques d'enseignant de mathématiques. In F. Vanderbrouck (Dir.), La classe de mathématiques : activité des élèves et pratiques enseignantes (pp. 59-68). Toulouse : Octares.

Roegiers, X. (2004). Une pédagogie de l'intégration : Compétences et intégration des acquis dans l'enseignement. Bruxelles : De Boeck.

Roegiers, X. (2010). La pédagogie de l'intégration: des systèmes d'éducation et de formation au cœur de nos sociétés. Bruxelles : De Boeck.

Roux-Perez, T. (2012). Construire la professionnalité enseignante à l'heure des réformes : enjeux de - et défis pour - la formation. In T. Pérez-Roux (Dir.), La professionnalité enseignante (pp. 97-120). Rennes : Presses Universitaires de Rennes.

Saujat, F. (2004). Comment les enseignants débutants entrent dans le métier. Formation et pratiques d'enseignement en questions, 1, 97-106.

Schmidt, M., \& Knowles, J.G. (1995). Four women's stories of « failure » as beginning teachers. Teaching and Teacher Education, 11(5), 429-444.

Tardif, J. (1998). La construction des connaissances. Les pratiques pédagogiques. Pédagogie collégiale, 11(3), 4-9.

Vergnaud, G. (1996). Au fond de l'action, la conceptualisation. In J.-M. Barbier (Dir.), Savoirs théoriques et savoirs d'action (pp. 275-292). Paris : PUF.

Vinatier, I. (2012). Les apports de l'analyse d'épisodes interactionnels problématiques pour de futurs enseignants en économie-gestion. In T. Pérez-Roux (Dir.), La professionnalité enseignante (pp. 41-56). Rennes : Presses Universitaires de Rennes.

Vinatier, I. (2013). Le travail enseignant. Une approche par la didactique professionnelle. Bruxelles : De Boeck. 


\section{RÉSUMÉS}

Cette étude vise à mieux comprendre l'autonomie du curriculum effectif au regard des prescriptions dont il est l'objet. Son objectif est de décrire ce qui oriente et guide l'activité de quatre étudiants-stagiaires d'EPS en formation par alternance afin d'identifier ce qu'ils font des recommandations curriculaires - notamment celles préconisant un enseignement par compétences. Les données recueillies croisent des informations issues d'enregistrements vidéo de séances de badminton (une par étudiant) et d'entretiens (ante séance, auto-confrontation simple et croisée). L'analyse a consisté à identifier les principes organisateurs de l'activité (Vinatier, 2013). Les résultats mettent en évidence trois préoccupations concomitantes mais hiérarchisées, au fondement de l'activité des étudiants-stagiaires lorsqu'ils sont aux prises avec le curriculum recommandé : construire sa posture d'enseignant ; susciter et maintenir les élèves dans l'activité; faire apprendre les élèves. Ils montrent que si les étudiants-stagiaires sont effectivement guidés par les recommandations, ils n'en retiennent que ce qui répond à leurs préoccupations.

This study aims to gain a better understanding of the possible discrepancy between the actual curriculum and its prescribed specifications. Its purpose is to describe what directs and guides the activity of four in-service training PE student teachers in order to identify how they deal with curriculum recommendations - including those promoting competency-based education. The data collected combine the video recordings of badminton lessons (one per student teacher) and three interviews (ante session, self- and peer-confrontation). The analysis consisted in identifying the organizational principles of the student teacher's activity (Vinatier, 2013). The findings highlight three associated but prioritised concerns that are the basis of the student teachers' activity: building their identity as a teacher; stimulating and maintaining the pupils' interest; promoting the pupils' learning. The analysis shows that even though student teachers may be guided by the curriculum recommendations, they tend to only select those that meet their concerns.

\section{INDEX}

Mots-clés : activité, étudiant-stagiaire, principes organisateurs, rapport au curriculum, éducation physique et sportive

Keywords : activity, student teachers, organizational principles, relationship to the curriculum, physical education

\section{AUTEUR}

\section{ELISABETH MAGENDIE}

Maître de conférences ESPE d'Aquitaine, équipe Vie Sportive du Laboratoire Cultures, Education, Société (LACES EA 4140), Université de Bordeaux 